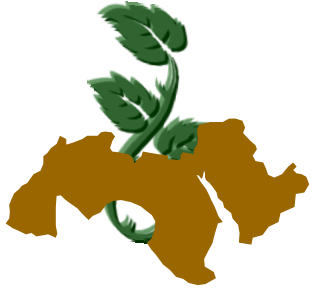

\title{
EFFECT OF PHOSPHORUS FERTILIZER TREATMENTS ON INCIDENCE OF FUSARIUM ROOT-ROT/WILT DISEASE COMPLEX, AND ON YIELD COMPONENTS OF LUPINE, CHICKPEA AND LENTIL CROPS
}

\author{
Rania Z. El-Shennawy'; M.M. Omran ${ }^{2}$ and F.A. Abd El-Motteleb ${ }^{3}$ \\ 1- Food Legume Disease, Section Plant Pathol. Res. Institute, ARC, Giza, Egypt \\ 2- Food Legumes Res. Section, Field Crops Res. Inst., ARC, Giza, Egypt \\ 3- Soils and Water Research Institute and Environment, Plant Nutrition Section, ARC, Giza, Egypt
}

Keywords: Phosphorus fertilizer, Wilt diseases, Legume crops

\section{ABSTRACT}

In greenhouse and field experiments, conducted in two successive winter seasons 2007/2009 the effect of phosphorus fertilizer treatments on incidence of wilt disease of lupine, chickpea and lentil, as well as yield and yield component was studied. Application of Phosphorus fertilizer (100 and $200 \mathrm{~kg} / \mathrm{fed}$; as super phosphate $\left(15.5 \% \mathrm{P}_{2} \mathrm{O}_{5}\right)$ before planting) caused a reduction in incidence and severity of wilt diseases on plants of the three legume crops either in greenhouse and field experiments. In field experiments, results indicated that the most effective treatment was phosphorus at 100 and $200 \mathrm{~kg} / \mathrm{fed}$ ) respectively, which reduced the wilt diseases. Phosphorus fertilizer significantly increased yield characters i.e., plant height, number of branches, number of pods, seeds yield / plant and weight of 100 seeds.

\section{INTRODUCTION}

Damping-off, root-rot and wilt diseases are destructive diseases attacking legume crops worldwide, causing serious yield losses. These diseases are caused by several fungi i.e., Rhizoctonia solani, Fusearium oxysporum, Fusarium solani and Sclerotium rolfsii (Abdallah, 1969; El-Garhy, 1994; Hassanein et al 1996 and Abou-Zeid et al 1997). Specific Fusarium oxysporum forma specials cause wilt on legume crops, i.e. F. oxysporum f.sp. lentis on lentil, F. oxysporum f.sp. ciceri on chick- pea and $F$. oxysporum f.sp. lupine on lupine (Booth, 1971). However, the major fungal disease problem of legume crops in Egypt is root-rot/wilt disease complex (Salem et al 1990 and El-Awadi et al 1990). The disease appears in most areas throughout the growing season in Egypt. Taha et al (1969) reported that Fusarium wilt caused by Fusarium oxysporum f.sp. ciceri is a major disease of chickpea.

Development of disease resistant varieties was the most economical mean of control. Phosphorus has been called "the key to life" because it is directly involved in most life processes. It exerts many and varied functions in plant metabolism and hence inadequate phosphate supply to the plant seriously affect numerous metabolic processes. Singh et al (1981) found that phosphorus applied at 60 and $90 \mathrm{~kg} / \mathrm{ha}$ significantly increased yields in comparison with $30 \mathrm{~kg} \mathrm{P}$, when 4 levels of $P(0$, 30,60 and $90 \mathrm{~kg} / \mathrm{ha}$ ) were applied. Negm et al (1992) reported that phosphorus application increased the number of branches and flowers per plant. Jain et al (1999) and Khurana and Sharma (2000) showed that the combined inoculation of Rhizobium and phosphate solubilizing bacteria increased nodulation, growth and yield parameters of chickpea. Increased germination, number of branches, nodulation and yield of pea and total biomass of chickpea compared with either individual inoculations or the uninoculated control (Rudresh et al 2005). This study was carried out in order to investigate the effect of phosphorus fertilizer on incidence of Fusarium root-rot / wilt disease complex and on yield component of lupine, chickpea and lentil crops. 


\section{MATERIALS AND METHODS}

This work has been carried out at Experimental Farm of Sers El-Layian Agricultural Research Station, Minufiya Governorate, Egypt. The mechanical and chemical properties of the experimental soil for the upper foot layer $(0-30 \mathrm{~cm})$ and available phosphorus of the experimental soil (Table 1) were determined before sowing in the two seasons according to the method described by Chapman and Pratt (1961) and presented in Table (1).

Mechanical and chemical properties of the experimental sites revealed that the texture of the soil was sandy clay with a low $\mathrm{P}$ content in the two growing seasons Table (1).

\section{Isolation and identification of pathogens}

Roots and basal stems of each of lupine, chickpea and lentil plants, showing typical symptoms of root-rot and wilt, were washed carefully with tap water, dried between two filter papers, then cut into small pieces. Pieces of infected roots were surface-sterilized in $3 \%$ sodium hypochlorite for 2 min., dried between two sterilized filter papers, then directly transferred to Petri dishes containing potato dextrose agar (PDA) medium, and incubated at $25^{\circ} \mathrm{C}$ for 5 days. The developed fungal hyphal were isolated, established in pure culture on PDA slants and finally purified by the single spore or hyphal tip techniques. The isolated fungal pathogens were identified as Fusarium oxysporum by the aid of Department of Mycology, Plant Pathology Institute, Agricultural Research Center, Giza, Egypt. Confirmation of pathogencity of these isolates was done on the host from which it was isolated. The pathogenicity tests revealed that these isolates were Fusairum oxysporum f.sp.

\section{Effect of phosphorus fertilizer treatments}

\section{Greenhouse Experiment}

Clay Pots $(25 \mathrm{~cm})$ were sterilized by dipping in $5 \%$ formation solution for $5 \mathrm{~min}$ and then left in open air till dryness. Soil sterilization was accomplished with $5 \%$ formalin solution, mixed thoroughly, covered with plastic sheet for one week and then the plastic sheet was removed in order to complete formalin evaporation for up to one month. Soil infestation with each individual Fusarium isolate was carried out at the rate of $5 \%$ of soil weight. Fungi were individually grown on sand-barley (SB) medium ( $25 \mathrm{~g}$ clean sand, $75 \mathrm{~g}$ barley and enough water to cover the mixture). Flasks contained sterilized medium were inoculated with each particular Fusarium isolate and incubated at $25^{\circ} \mathrm{C}$ for two weeks. Soil of control pots was mixed with the same amount of sterilized sand-barley (SB) medium. The phosphorus fertilizer rates $(0,100$ and $200 \mathrm{~kg} / \mathrm{fa}$ ) was mixed with the top layer of the soil before sowing. Ten seeds of lentil (Giza 9), chickpea (Giza 3) and lupine (Giza 1) were sown in each pot, and three replicates were used for each treatment. Percentages of pre- and postemergence damping-off as well as healthy survival plants in each treatment were determined 15 and 30 days after sowing, respectively using the next formula according to El-Helaly et al (1970).

Disease severity: It was estimated using the following equation.

Disease severity $=[(a \times b \div N \times K) \times 100]$ (Soleman et al 1988).

a: Number of infected plants.

b: Grade of infection.

$\mathrm{N}$ : Number of total plants.

$\mathrm{K}$ : Maximum grade of infection

Infection types (IT): roots were rated for disease severity according to the scale adopted by Aegerter et al (2000) as follows:

0 : no symptoms.

1 : few lesions (covering $<10 \%$ of root), secondary root rot slight.

2 : rot of secondary roots or lesions covering approximately $25 \%$ of the root.

3 : lesions covering at least $50 \%$ of the root and dead secondary roots.

4: general root rot, most of the root affected, also including (pre- and post-emergence dampingoff).

\section{Field experiments}

Two field experiments were carried out in the Experimental Farm of Sers El-Layian, Agricultural Research Station, Minufiya Governorate, Egypt, during two winter seasons (2007/2008 and 2008/2009). Each experiment included three legume crops, i.e. lupine, chick-pea and lentil in the main plots, without application and foliar application of the foliar spray treatments were: a) control (spraying with water) and b) foliar spraying with phosphorus solution fertilizer in the split plots. Extraction solution of phosphorus fertilizer was prepared by dissolved calcium superphosphate 15.5\% $\mathrm{P}_{2} \mathrm{O}_{5}$ as a rate of $50 \mathrm{~g} /$ /iter in the stock pot then flipping exactly and leave it at 48 hours and look the solution after precipitation, filter through any good grade phosphate-free filter paper. Taked an a filtering solution for used in spraying the plants at the rate of 400 liter/feddan. The plants were 
sprayed twice at i.e., after 35 and 75 days of planting respectively. Phosphorus fertilizer rates $(0,100$ and $200 \mathrm{~kg} / \mathrm{fa}$ ) in the splite-split plots. For each experiment, a split-split plot design with three replicates was followed. The plot size was $6 \mathrm{~m}^{2}$ consisting of three rows, each row 3 meter long spaced at $60 \mathrm{~cm}$. In case of Lupine, two seeds were drilled in one side of rows, and the spacing between hills was $20 \mathrm{~cm}$. In case of Chickpea, two seeds were dilled in the two sides of rows, the spacing between hills was $10 \mathrm{~cm}$, hen then thinning took place of janumery leaving one plant / hill in a chickpea. In case of lentil: the seeds were drilled in the two sides of each row.

The rates of phosphors fertilization $(0,100$ and $200 \mathrm{~kg} / \mathrm{fa}$ ) were applied as super phosphate $\left(15.5 \% \mathrm{P}_{2} \mathrm{O}_{5}\right)$ before planting. The nitrogenous fertilizer at the rate of $15 \mathrm{~kg}$ per feddan was applied before the first irrigation as a starter dose. The first foliar application of phosphorus fertilizer was applied after 35 days from planting and the second application was applied at the first flowering in after 35 and 75 days from sowing. At maturity, plants were harvested and the following yield characters, i.e. plant height, number of branch, number of pods / plant, seeds weight / plant, 100seeds / weight as well as seeds, straw and total yields / fad were recorded,

Seed samples of each crop were taken and dried, then ground to powder for chemical analysis. Nitrogen content was determined using improving macro-kjeldahl method (A.O.A.C., 1975). The percentage of seed protein content was calculated by multiplying the percentage of nitrogen content by the factor of 6.25 according to Tripathi et al (1971). Phosphorus content was determined calorimetrically using spectro-photomater was determined using flame photometer according to Chapman and Pratt (1961)

\section{Statistical Analysis}

All collected data were subjected to statistical analysis for each season and to combined analysis over years according to Gomez and Gomez (1984).

\section{RESULTS}

\section{Effect of Phosphorus Fertilizers in Greenhouse}

Data in Table (2) illustrate the effect of super phosphate $(0,100,200 \mathrm{~kg} / \mathrm{fed})$ on the percentage of pre- and post-emergence damping-off, wilt dis- ease severity caused by the fungal pathogens, Fusairum oxysporum f.sp. All tested phosphorus fertilizers significantly reduced the development of root-rot disease, under greenhouse conditions compared with control. The highest percentage of survival plant in all legume crops was resulted in soil fertilized with super phosphate at $200 \mathrm{~kg} / \mathrm{fed}$ ).

\section{Effect of Phosphorus Fertilizers in the field}

\section{Effects on disease incidence}

Data in Table (3) clearly show the influence of super phosphate $(0,100$ and $200 \mathrm{~kg} / \mathrm{fed})$ on preand post-emergence damping-off of lupine, chickpea and lentil under field conditions during two successive seasons. The results revealed that application of both fertilizer rates (100 and 200 $\mathrm{kg} / \mathrm{fed}$ ) significantly reduced pre- and postemergence damping-off compared to un-treated plants (control). In general, the protection degree offered by super phosphate fertilizer applied at rate of $200 \mathrm{~kg} / \mathrm{fed}$ was much higher than untreated control.

\section{Effects on plant growth characters}

The obtained data in Table (4) revealed a significant effect of fertilization with super phosphate over the control where these effects were shown in case of plant height, fresh weight and dry weight. The obtained results were confirmed from the two experiments conducted in 2007/2008 and $2008 / 2009$ seasons, respectively. However, the great effect on plant growth was given by fertilization with super phosphate $(200 \mathrm{~kg} / \mathrm{fed})$ application compared with non-fertilized control.

\section{Effect of foliar spraying of lupine, chickpea and} lentil plants with super phosphate

\section{Effects on severity of root-rot/wilt}

Data in Table (5) show the efficacy of spraying super phosphate on root-rot/wilt disease severity under field conditions in two seasons, 2007/2008 and 2008/2009. The obtained results revealed that the super phosphate spray reduced significantly disease severity of $F$. oxysporum compared to untreated one (control). The protection effect was increased by increasing of spraying number. The plants received 3 sprays showed the lowest disease severity. 
Table 2. Effect of super phosphate, on incidence and severity of root-rot/wilt disease complex of lupine, chickpea and lentil plants, grown in soil infested with specific forma species of Fusarium oxysporum, under greenhouse conditions.*

\begin{tabular}{|c|c|c|c|c|c|c|}
\hline Crop & $\begin{array}{l}\text { Treatment } \\
\text { kg/fed }\end{array}$ & Fungus & $\begin{array}{c}\text { Pre- } \\
\text { emergence } \\
\text { damping-off \% }\end{array}$ & $\begin{array}{c}\text { Post- } \\
\text { emergence } \\
\text { damping-off \% }\end{array}$ & $\begin{array}{c}\text { Survived } \\
\text { plant } \\
\%{ }^{\star \star} \\
\end{array}$ & $\begin{array}{c}\text { Disease } \\
\text { severity } \\
\%{ }^{\star \star \star} \\
\end{array}$ \\
\hline \multirow{4}{*}{ Lupine } & 0 & \multirow{4}{*}{$\begin{array}{l}\text { F. oxysporum } \\
\text { f.sp. lupini }\end{array}$} & 23.33 & 20.0 & 56.67 & 61.67 \\
\hline & 100 & & 13.33 & 13.33 & 73.34 & 45.00 \\
\hline & 200 & & 10.00 & 6.66 & 83.34 & 41.67 \\
\hline & Mean & & 15.55 & 13.33 & 71.12 & 49.45 \\
\hline \multirow{4}{*}{ Chickpea } & 0 & \multirow{4}{*}{$\begin{array}{l}\text { F. oxysporum } \\
\text { f.sp. ciceri }\end{array}$} & 13.33 & 23.33 & 63.34 & 68.33 \\
\hline & 100 & & 6.66 & 20.00 & 73.34 & 51.67 \\
\hline & 200 & & 0.00 & 10.00 & 90.00 & 36.67 \\
\hline & Mean & & 6.66 & 17.78 & 75.56 & 52.22 \\
\hline \multirow{4}{*}{ Lentil } & 0 & \multirow{4}{*}{$\begin{array}{c}\text { F. oxysporum } \\
\text { f.sp. lentis }\end{array}$} & 23.33 & 26.66 & 50.00 & 65.00 \\
\hline & 100 & & 16.66 & 13.33 & 70.00 & 46.67 \\
\hline & 200 & & 13.33 & 3.33 & 83.33 & 30.00 \\
\hline & Mean & & 17.77 & 14.44 & 67.78 & 47.22 \\
\hline \multicolumn{2}{|c|}{ L.S.D. at 0.5} & & 8.51 & 8.43 & 12.58 & 6.97 \\
\hline
\end{tabular}

* The pathogens used were: Fusairum oxysporum f.sp. lentis on lentil, F. oxysporum f.sp. ciceri on chickpea and

F. oxysporum f.sp. lupine on lupine.

** After 30 days from sowing.

*** Determined after 60 days from sowing.

Table 3. Effect of different rates $(0,100$ and $200 \mathrm{~kg} / \mathrm{fed})$ of super phosphate soil fertilizer on incidence of root-rot/wilt disease of lupine, chickpea and lentil during two seasons under field conditions

\begin{tabular}{|c|c|c|c|c|c|c|c|}
\hline \multirow{3}{*}{ Crop } & \multirow{3}{*}{$\begin{array}{l}\text { Treatment } \\
\text { kg/fed }\end{array}$} & \multicolumn{6}{|c|}{ Disease incidence } \\
\hline & & \multicolumn{3}{|c|}{ Season $2007-2008$} & \multicolumn{3}{|c|}{ Season 2008-2009 } \\
\hline & & Pre-\% & Post-\% & Survival \% & Pre-\% & Post-\% & Survival \% \\
\hline \multirow{3}{*}{ Lupine } & 0 & 20.0 & 16.67 & 63.33 & 23.33 & 20.00 & 56.67 \\
\hline & 100 & 16.67 & 10.00 & 73.33 & 13.33 & 3.33 & 83.33 \\
\hline & 200 & 6.67 & 3.33 & 90.00 & 3.33 & 0.00 & 56.67 \\
\hline Mean & - & 14.45 & 10.00 & 75.55 & 13.33 & 7.78 & 65.55 \\
\hline \multirow{3}{*}{ Chickpea } & 0 & 20.0 & 10.00 & 70.00 & 20.00 & 23.33 & 56.67 \\
\hline & 100 & 13.3 & 3.33 & 83.33 & 16.67 & 10.00 & 73.33 \\
\hline & 200 & 3.33 & 0.00 & 96.66 & 3.33 & 3.33 & 93.33 \\
\hline Mean & - & 12.22 & 4.44 & 83.33 & 13.33 & 12.22 & 74.44 \\
\hline \multirow{3}{*}{ Lentil } & 0 & 20.0 & 13.33 & 66.67 & 26.67 & 26.67 & 46.67 \\
\hline & 100 & 13.33 & 10.00 & 76.67 & 20.00 & 13.33 & 66.67 \\
\hline & 200 & 3.33 & 6.67 & 900.0 & 6.67 & 6.67 & 86.67 \\
\hline Mean & - & 12.22 & 10.0 & 77.78 & 17.78 & 15.55 & 66.67 \\
\hline \multicolumn{8}{|l|}{ L.S.D. at 0.05: } \\
\hline Crops A & & 5.41 & 3.95 & 6.55 & 5.23 & 5.41 & 9.47 \\
\hline Treatment B & & 7.34 & 7.96 & 9.75 & 7.96 & 5.03 & 12.34 \\
\hline Interaction $\mathrm{AB}$ & & 9.90 & 7.38 & 11.43 & 8.73 & 8.08 & 15.12 \\
\hline
\end{tabular}


Table 4. Effect of different rates $(0,100$ and $200 \mathrm{~kg} / \mathrm{fed})$ of super phosphate on some growth characters of lupine, chickpea and lentil, grown during two seasons under field conditions

\begin{tabular}{|c|c|c|c|c|c|c|c|}
\hline \multirow[b]{3}{*}{ Crop } & \multirow[b]{3}{*}{$\begin{array}{c}\text { Treatment } \\
\mathrm{kg} / \mathrm{fed}\end{array}$} & \multicolumn{6}{|c|}{ Crop parameters of (lupine, chickpea and lentil) plant during } \\
\hline & & \multicolumn{3}{|c|}{ Season 2007-2008 } & \multicolumn{3}{|c|}{ Season $2008-2009$} \\
\hline & & $\begin{array}{l}\text { Plant } \\
\text { height } \\
(\mathrm{cm})\end{array}$ & $\begin{array}{c}\text { Fresh } \\
\text { weight/plant } \\
\text { (g) }\end{array}$ & $\begin{array}{c}\text { Dry } \\
\text { weight/plant } \\
\text { (g) }\end{array}$ & $\begin{array}{c}\text { Plant } \\
\text { height }(\mathrm{cm})\end{array}$ & $\begin{array}{c}\text { Fresh } \\
\text { weight/plant } \\
\text { (g) }\end{array}$ & $\begin{array}{c}\text { Dry } \\
\text { weight/plant } \\
\text { (g) }\end{array}$ \\
\hline \multirow{3}{*}{ Lupine } & 0 & 32.67 & 10.33 & 0.97 & 28.67 & 9.83 & 1.13 \\
\hline & 100 & 34.33 & 10.73 & 1.17 & 31.00 & 10.10 & 1.50 \\
\hline & 200 & 34.33 & 11.43 & 1.43 & 32.33 & 11.53 & 1.83 \\
\hline \multirow[t]{2}{*}{ Mean } & - & 33.77 & 10.83 & 1.19 & 30.67 & 10.48 & 1.49 \\
\hline & 0 & 22.67 & 7.30 & 0.68 & 20.33 & 7.07 & 0.77 \\
\hline \multirow[t]{2}{*}{ Chickpea } & 100 & 25.00 & 7.50 & 0.77 & 23.33 & 7.77 & 0.70 \\
\hline & 200 & 36.00 & 8.47 & 0.82 & 25.00 & 8.60 & 0.93 \\
\hline \multirow[t]{2}{*}{ Mean } & - & 27.89 & 7.75 & 0.75 & 22.89 & 7.81 & 0.80 \\
\hline & 0 & 17.67 & 4.33 & 0.56 & 14.33 & 4.33 & 0.62 \\
\hline \multirow[t]{2}{*}{ Lentil } & 100 & 20.67 & 4.83 & 0.62 & 16.00 & 5.23 & 0.67 \\
\hline & 200 & 22.00 & 5.57 & 0.63 & 17.67 & 6.00 & 0.77 \\
\hline Mean & - & 20.11 & 4.91 & 0.60 & 8.54 & 5.18 & 0.68 \\
\hline \multicolumn{8}{|c|}{ L.S.D. at 0.05 : } \\
\hline \multicolumn{2}{|c|}{ Crops A } & 0.76 & 0.26 & 0.090 & 0.86 & 0.45 & 0.15 \\
\hline \multicolumn{2}{|l|}{ Treatment B } & 1.02 & 0.19 & 0.14 & 1.18 & 0.39 & 0.12 \\
\hline \multicolumn{2}{|c|}{ Interaction $\mathrm{AB}$} & 1.55 & 0.46 & 0.18 & 1.40 & 0.75 & 0.22 \\
\hline
\end{tabular}

Table 5. Effect of interaction between fertilization and spraying super phosphate on severity of wilt disease on lupine, chickpea and lentil foliages, under field conditions.*

\begin{tabular}{|c|c|c|c|c|c|c|c|c|}
\hline \multirow{2}{*}{ Crop } & \multirow{2}{*}{ Spray } & \multirow{2}{*}{$\begin{array}{c}\text { Treatment } \\
\text { kg/fed }\end{array}$} & \multicolumn{3}{|c|}{$2007 / 2008$} & \multicolumn{3}{|c|}{$2008 / 2009$} \\
\hline & & & $1^{\star \star}$ & $2^{\star \star \star}$ & Mean & 1 & 2 & mean \\
\hline \multirow{6}{*}{ Lupine } & \multirow{3}{*}{$\begin{array}{c}\text { No } \\
\text { spray }\end{array}$} & 0 & 18.68 & 19.77 & 19.22 & 22.96 & 25.18 & 24.07 \\
\hline & & 100 & 17.00 & 18.20 & 17.60 & 20.00 & 22.88 & 21.41 \\
\hline & & 200 & 15.55 & 17.00 & 10.85 & 18.88 & 22.22 & 20.55 \\
\hline & \multirow{3}{*}{ Spray } & 0 & 13.15 & 13.70 & 13.42 & 15.18 & 15.52 & 15.35 \\
\hline & & 100 & 12.00 & 12.87 & 12.43 & 12.59 & 13.33 & 12.96 \\
\hline & & 200 & 10.37 & 11.93 & 11.15 & 11.11 & 11.70 & 11.40 \\
\hline \multirow{6}{*}{ Chickpea } & \multirow{3}{*}{$\begin{array}{c}\text { No } \\
\text { spray }\end{array}$} & 0 & 20.74 & 22.22 & 21.48 & 25.45 & 27.58 & 26.51 \\
\hline & & 100 & 18.34 & 19.83 & 19.08 & 23.70 & 26.66 & 25.18 \\
\hline & & 200 & 16.40 & 16.93 & 33.33 & 20.74 & 23.48 & 22.11 \\
\hline & \multirow{3}{*}{ Spray } & 0 & 17.33 & 18.66 & 17.99 & 21.48 & 22.48 & 21.98 \\
\hline & & 100 & 15.33 & 16.17 & 15.75 & 20.00 & 20.33 & 20.16 \\
\hline & & 200 & 14.27 & 15.33 & 14.80 & 18.00 & 18.59 & 18.29 \\
\hline \multirow{6}{*}{ Lentil } & \multirow{3}{*}{$\begin{array}{c}\text { No } \\
\text { spray }\end{array}$} & 0 & 21.11 & 22.15 & 21.63 & 33.33 & 35.00 & 34.16 \\
\hline & & 100 & 17.00 & 18.10 & 17.55 & 30.00 & 32.22 & 31.11 \\
\hline & & 200 & 16.00 & 16.87 & 16.43 & 28.88 & 30.00 & 29.44 \\
\hline & \multirow{3}{*}{ Spray } & 0 & 16.40 & 17.17 & 16.78 & 20.37 & 20.74 & 20.55 \\
\hline & & 100 & 13.76 & 15.17 & 14.46 & 20.00 & 20.00 & 20.00 \\
\hline & & 200 & 12.33 & 13.48 & 12.90 & 17.77 & 18.88 & 18.32 \\
\hline
\end{tabular}

L.S.D. at 0.05:

$\begin{array}{lllll}\text { Crops } & 0.27 & 0.21 & 0.38 & 0.42 \\ \text { Spray } & 0.41 & 0.10 & 0.46 & 0.40 \\ \text { Treatment } & 0.36 & 0.18 & 0.56 & 0.61 \\ \text { Interaction } & 0.74 & 0.46 & 1.00 & 1.03\end{array}$

* Foliar spray applied at rate.

** The first spray was applied after 35 from sowing.

${ }^{\star * \star}$ Further additional spray applied after 75 from sowing. 


\section{Effects on growth characters and yield compo- nents}

Data presented in Table (6) showed that all treatments gave high values of plant height and number of branches. Super phosphate spray treatment was more effective on these parameters. Marked differences were detected among all treatments on yield components of lupine, chickpea and lentil plants. Lupine recorded the highest values of number of pods/plant and weight of 100seed, the application of super phosphate spray increased significantly number of pods/plant and weight of 100-seed in all crops. On the other hand, no spray had any significant effect in number of pods/plant but increased weight of 100 -seed.
Effect of super phosphate fertilizers on chemical composition of lupin, chickpea and lentil plants

The present results indicated that the different rates of super phosphates applied to soils or with additional foliar spray caused significant increase in soluble and total nitrogen contents in lupine, chickpea and lentil plants.

The present results in Table (7) are agreed with those obtained by Mengle and Krikby (1987). They found that in seeds and grains, $\mathrm{P}$ contents in the range of 0.4 to $0.5 \%$ in the dry matter. However, data indicated that the root-rot diseases of (lupine, chickpea and lentil) plants were decreased with $\mathrm{P}$ fertilization Tables (7).

Table 6. Effect of super phosphate spray on some yield component of lupin, chickpea and lentil under field conditions

\begin{tabular}{|c|c|c|c|c|c|c|c|c|c|c|c|c|}
\hline \multirow[b]{3}{*}{ Crop } & \multirow[b]{3}{*}{ Spray } & \multirow{3}{*}{ 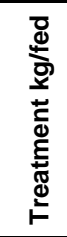 } & \multicolumn{10}{|c|}{ Season } \\
\hline & & & \multicolumn{5}{|c|}{$2007 / 2008$} & \multicolumn{5}{|c|}{ 2008/2009 } \\
\hline & & & $\begin{array}{l}\text { Plant } \\
\text { height } \\
(\mathbf{c m})\end{array}$ & $\begin{array}{l}\text { Branch- } \\
\text { es plant }\end{array}$ & $\begin{array}{l}\text { No. } \\
\text { seeds } \\
\text { pods }\end{array}$ & $\begin{array}{c}100- \\
\text { seed } \\
\text { weight } \\
(\mathrm{g})\end{array}$ & $\begin{array}{c}\text { Seed } \\
\text { weight } \\
\text { ardab/ } \\
\text { fed. }\end{array}$ & $\begin{array}{l}\text { Plant } \\
\text { height }\end{array}$ & $\begin{array}{l}\text { Branch- } \\
\text { es/plant }\end{array}$ & $\begin{array}{l}\text { No. } \\
\text { seeds } \\
\text { pods }\end{array}$ & $\begin{array}{c}100- \\
\text { seed } \\
\text { weight } \\
\text { (g) }\end{array}$ & $\begin{array}{c}\text { Seed } \\
\text { weight } \\
\text { ardab/ } \\
\text { fed. }\end{array}$ \\
\hline \multirow{6}{*}{ Lupine } & \multirow{3}{*}{$\begin{array}{c}\text { No } \\
\text { spray }\end{array}$} & 0 & 108.00 & 2.00 & 23.33 & 28.00 & 3.44 & 99.00 & 2.00 & 18.00 & 28.67 & 3.70 \\
\hline & & 100 & 113.33 & 2.66 & 24.33 & 29.00 & 5.25 & 103.33 & 2.66 & 19.00 & 31.67 & 4.87 \\
\hline & & 200 & 115.00 & 3.00 & 25.33 & 31.67 & 5.66 & 105.00 & 3.00 & 21.00 & 32.67 & 5.51 \\
\hline & \multirow{3}{*}{ Spray } & 0 & 127.66 & 3.00 & 24.33 & 31.67 & 4.23 & 121.67 & 3.00 & 22.00 & 29.00 & 5.89 \\
\hline & & 100 & 129.66 & 3.66 & 25.00 & 34.33 & 6.27 & 123.33 & 3.66 & 23.00 & 34.00 & 6.59 \\
\hline & & 200 & 135.00 & 4.00 & 30.67 & 36.33 & 7.07 & 128.33 & 4.00 & 25.00 & 36.33 & 7.71 \\
\hline \multirow{6}{*}{ Chickpea } & \multirow{3}{*}{$\begin{array}{c}\text { No } \\
\text { spray }\end{array}$} & 0 & 79.67 & 1.66 & 39.33 & 21.33 & 3.07 & 73.33 & 2.33 & 43.67 & 23.67 & 3.31 \\
\hline & & 100 & 81.33 & 1.66 & 41.67 & 23.00 & 4.42 & 76.66 & 3.33 & 45.00 & 25.33 & 3.75 \\
\hline & & 200 & 88.00 & 2.00 & 44.33 & 24.33 & 5.04 & 83.33 & 3.66 & 48.67 & 27.00 & 4.50 \\
\hline & \multirow{3}{*}{ Spray } & 0 & 88.33 & 3.33 & 48.00 & 25.67 & 4.09 & 83.33 & 4.00 & 50.00 & 28.67 & 3.60 \\
\hline & & 100 & 91.62 & 3.33 & 49.33 & 26.67 & 5.69 & 88.00 & 4.00 & 54.00 & 30.00 & 5.42 \\
\hline & & 200 & 95.00 & 3.66 & 53.67 & 28.67 & 6.14 & 90.00 & 4.33 & 55.00 & 31.00 & 5.88 \\
\hline \multirow{6}{*}{ Lentil } & \multirow{3}{*}{$\begin{array}{c}\text { No } \\
\text { spray }\end{array}$} & 0 & 60.67 & 3.00 & 18.67 & 4.33 & 2.30 & 51.67 & 5.33 & 24.00 & 5.33 & 2.64 \\
\hline & & 100 & 61.67 & 3.00 & 21.33 & 5.33 & 3.06 & 55.00 & 5.33 & 27.00 & 6.00 & 3.01 \\
\hline & & 200 & 65.00 & 3.66 & 23.67 & 5.66 & 3.93 & 58.00 & 6.33 & 29.33 & 7.00 & 3.60 \\
\hline & \multirow{3}{*}{ Spray } & 0 & 63.00 & 3.66 & 26.00 & 5.00 & 3.53 & 63.67 & 6.66 & 32.00 & 8.33 & 3.47 \\
\hline & & 100 & 69.33 & 4.66 & 30.67 & 6.67 & 4.39 & 61.67 & 7.00 & 36.00 & 8.67 & 4.89 \\
\hline & & 200 & 77.67 & 5.33 & 33.33 & 7.33 & 5.13 & 65.00 & 7.00 & 39.33 & 9.00 & 5.94 \\
\hline
\end{tabular}

L.S.D. at 0.05:

$\begin{array}{lllllllllll}\text { Crops } & 1.41 & 0.22 & 0.56 & 0.41 & 0.72 & 1.65 & 0.21 & 0.68 & 0.44 & 0.16 \\ \text { Spray } & 1.34 & 0.47 & 0.43 & 0.42 & 0.45 & 1.40 & 0.47 & 0.56 & 0.52 & 0.27 \\ \text { Treatment } & 3.25 & 0.36 & 1.71 & 1.09 & 1.09 & 2.19 & 0.43 & 1.19 & 1.47 & 0.33 \\ \text { Interaction } & 3.82 & 0.75 & 1.64 & 1.49 & 1.64 & 3.95 & 0.71 & 1.75 & 1.47 & 0.50\end{array}$

* Foliar spray applied at rate of 100 liter / 5 kg super phosphate.

** The first spray was applied after 75 from sowing.

*** Further additional spray applied after 95 from sowing. 
Table 7. Effect of super phosphate treatments on chemical composition of lupin, chickpea and lentil plants

\begin{tabular}{|c|c|c|c|c|c|c|c|c|}
\hline \multirow{2}{*}{ Crop } & \multirow{2}{*}{ Spray* } & \multirow{2}{*}{ Concentration } & \multicolumn{3}{|c|}{$2007 / 2008$} & \multicolumn{3}{|c|}{$2008 / 2009$} \\
\hline & & & $\mathrm{P} \%$ & $\mathrm{~N} \%$ & Protein \% & P\% & N\% & Protein \% \\
\hline \multirow{6}{*}{ Lupin } & \multirow{3}{*}{$\begin{array}{c}\text { no. } \\
\text { spray }\end{array}$} & 0 & 0.30 & 3.69 & 23.07 & 0.33 & 3.79 & 23.66 \\
\hline & & 100 & 0.36 & 3.94 & 24.76 & 0.37 & 4.05 & 25.31 \\
\hline & & 200 & 0.46 & 4.20 & 26.26 & 0.45 & 4.18 & 26.14 \\
\hline & \multirow{3}{*}{ Spray } & 0 & 0.38 & 3.77 & 23.55 & 0.39 & 3.91 & 24.43 \\
\hline & & 100 & 0.56 & 4.15 & 25.89 & 0.57 & 4.22 & 26.40 \\
\hline & & 200 & 0.59 & 4.49 & 28.04 & 0.62 & 4.49 & 28.09 \\
\hline \multirow{6}{*}{ Chickpea } & \multirow{3}{*}{$\begin{array}{c}\text { no } \\
\text { spray }\end{array}$} & 0 & 0.47 & 3.57 & 22.35 & 0.46 & 3.72 & 23.23 \\
\hline & & 100 & 0.53 & 3.95 & 24.71 & 0.55 & 4.01 & 25.08 \\
\hline & & 200 & 0.55 & 4.09 & 25.55 & 0.61 & 4.16 & 26.01 \\
\hline & \multirow{3}{*}{ Spray } & 0 & 0.56 & 3.83 & 23.93 & 0.58 & 3.88 & 24.29 \\
\hline & & 100 & 0.60 & 4.09 & 25.58 & 0.65 & 4.15 & 25.9 \\
\hline & & 200 & 0.63 & 4.24 & 26.53 & 0.67 & 4.28 & 26.77 \\
\hline \multirow{6}{*}{ Lentil } & \multirow{3}{*}{$\begin{array}{c}\text { no } \\
\text { spray }\end{array}$} & 0 & 0.37 & 3.47 & 21.72 & 0.39 & 3.56 & 22.29 \\
\hline & & 100 & 0.44 & 3.74 & 23.42 & 0.44 & 3.79 & 23.71 \\
\hline & & 200 & 0.58 & 4.02 & 25.16 & 0.55 & 4.07 & 25.44 \\
\hline & \multirow{3}{*}{ Spray } & 0 & 0.42 & 3.75 & 23.45 & 0.42 & 3.78 & 23.66 \\
\hline & & 100 & 0.55 & 3.95 & 24.70 & 0.61 & 4.03 & 25.23 \\
\hline & & 200 & 0.61 & 4.12 & 25.95 & 0.64 & 4.25 & 26.54 \\
\hline
\end{tabular}

* Plants were sprayed twice.

\section{DISCUSSION}

The results of this study indicated that resistance of (lupine, chickpea and lentil) against root-rot and wilt could be induced by super phosphate. In greenhouse and field condition, super phosphate treatments significantly reduced preand post-emergence damping-off as well as rootrot disease, consequently increased germination percentage and healthy plants. In addition, super phosphate treatment increased significantly vegetative growth parameters and yield component. Fungal infection is considered as one of the most important stress conditions. Such enhancement effect of super phosphate on the vegetative growth parameters might be attributed to its effect on nodulation and yield parameters (Jain et al 1999; Khurana and Sharma, 2000 and Rudresh et al 2005). They added that super phosphate is required for the synthesis of tryptophane which, consider the precursor of IAA. The formation of such growth substances is also due to indirect effect of by super phosphate treatment.

Data presented in Table (5) showed that the soil application of phosphorus fertilizer increased plant height, number of branches / plant, number of pods / plant, 100-seed weight / g) and seed weight ardab / fed in both seasons of the three legume varieties. It is clear from the same table that foliar spraying of phosphorus solution significantly increased all these parameters. These results are in agreement with (Ahmed et al 1992), they found that the application $P$ increased significantly protein content and carbohydrate content in seed and different plant parts.

The interaction effect of foliar spraying of phosphorus solution and the soil application as a rate $200 \mathrm{~kg} /$ fed calcium superphosphate $15.5 \mathrm{P}_{2} \mathrm{O}_{5}$ gave the highest values in the all legume varieties. These results are agreed with those obtained by Singh et al (1981). They found that, $P$ applied at 60 and $90 \mathrm{~kg} /$ ha significantly increased yields in comparison with $30 \mathrm{~kg} /$ ha $\mathrm{P}$, when 4 levels of $\mathrm{P}$ (0, 30, 60 and $90 \mathrm{~kg} / \mathrm{ha})$ were applied. ElGharably and Abd El-Razek (1982) studied the effect of various levels of $P$ being added to a sandy calcareous soil on snap been grown in greenhouse for 35 days. They found that the maximum growth was obtained by 150 ppm $P$ application. Negm et al (1992) observed that, phosphorus application increased the number of branches and flowers per plant

It is obvious from these data that all intensive systems of arable cropping have a relatively high 
demand for phosphate. Phosphorus is particularly important for leguminous plants possibly by its influence on the activity of the rhizobioum bacteria. For mixed swords it is therefore important that soil $P$ levels should be kept high in order to maintain the leguminous species. (Mengle and Krikby, 1987). Also, phosphorus exerts many and varied functions in plants metabolism and hence inadequate phosphate supply to the plant seriously affects numerous metabolic processes. It is well shown that, the most important function of $P$ formation of pyrophosphate bonds allow energy transfer (Mengle and Krikby, 1987).

\section{REFERENCES}

Abdallah, A.M.A. (1969). Studies on Root Disease of Broad Bean in Egypt. pp. 45-48, M.Sc. Thesis, Fac. Agric., Ain Shams Univ., Cairo, Egypt. Abou-Zeid, N. M.; G. A. El-Morsy; A. M. Hassanein and M. K. Arafa (1997). Identification of fusarium wilt caused by Fusarium oxysporum and pathogen variability in faba bean, lentil and chickpea crops in Egypt. J. Agric. Res., 75(3): 529541.

Aegerter, B.J.; T.R. Gordon and R.M. Davis, (2000). Occurrence and pathogenicity of fungi associated with melon root-rot and vine decline in California. Plant Dis., 84: 224-230.

Ahmed, S.A.; F.A. Abdel-Mottaleb; S.A. Ismail, and F.H. El-Gendi, (1992). Response of brood bean (Vicia faba L.) to application of phosphorus and zinic. Egypt J. Appl. Sci., 7(11): 418 - 426.

A.O.A.C (1975). Association of Official Agricultural Chemists. Official Methods of Analysis. $12^{\text {th }} \mathrm{Ed}$. p. 832. AOAC, Washington DC.

Booth, C. (1971). The Genus Fusarium. p. 237. CNU, Kew, Surrey, England,

Chapman, H.D. and J.F. Pratt, (1961). Methods of Analysis for Soils Plants and Waters. pp. 201-203. Division of Agricultural Science, Univ. of California, Barkely. USA.

El-Awadi, F.A.; I.A.I. Smail and E.A. Nadia, (1990). Evaluation of lupine entries and fungicides against root rot and wilt diseases of lupine, Proc. $8^{\text {th }}$ Congress of the Egyptian Phytopathol. Soc., Cairo, pp. 393-395.

El-Garhy, A.M. (1994). Studies on Root-rot and Wilt Diseases of Alfalfa. p. 128. M.Sc. Thesis, Fac. of Agric., El-Azhar Univ., Cairo, Egypt.

El-Gharably, G.A. and M. Abd El-Razek, (1982). Response of snap beans to applications of $P$ and $\mathrm{Fe}$ in calcareous soil. Journal of Plant Nutrition, 5: 721-728.

El-Helaly, A.F.; H.M. Elarosi; M.W. Assawah, and M.T. Abol-Wafa, (1970). Studies on damping- off and root-rots of bean in UAR (Egypt). Egypt. J. Phytopathol., 2: 41-57.

Gomez, K.A. and A.A. Gomez (1984). Statistical Procedures for Agricultural Research. pp. 424452. John Willy and Sons. Inc. New York.

Hassanein, A.M.; G.A. El-Morsy and N.M. AbouZeid, (1996). Biological control of damping-off, root-rot and wilt diseases of lentil. Proceedings du Symposium Regional sur les Maladies des cereals et des Legumineuses, pp. 369-374. 1114 November 1996 Rabat, Maroc.

Jain, P.C.; P.S. Kushawaha; U.S. Dhakal; H. Khan and S.M. Trivedi, (1999). Response of chickpea (Cicer arietinum L.) to phosphorus and biofertilizer, Legume Res., 22: 241-244.

Khurana, A.S. and P. Sharma, (2000). Effect of dual inoculation of phosphate solubilizing bacteria, Bradyrhizobium sp. (cicer) and phosphorus on nitrogen fixation on yield of chickpea. Indian $\mathbf{J}$. Pulses Res., 13: 66-67.

Mengel, K. and E.A. Krikby, (1987). Principles of Plant Nutrition $4^{\text {th }}$ Ed. pp. 40-42. Publisher International Potash. Institutes P.O. Box CH 3038 Worblaufen, Bern, Switzerland.

Negm, M.A.; M.A. Abdel-Rehem and S.Y. Montasser (1992). Effect of different rates and frequencies of foliar applications of phosphorus on sunflower growing on calcareous soils. Egypt. J. Soil Sci., 32: 513-521.

Rudresh, D. L.; M. K. Shivaprakash, and R. D. Prasad (2005). Effect of combined application of Rhizobium, phosphate solubilzing bacterium and Trichoderma spp. on growth, nutrient uptake and yield chicpea (Cicer arietinum L.). Applied Soil Ecology, 28: 139-146.

Salem, D.S.; E.A. Omar and A.M. Khattab (1990). Screening for resistance to root-rot wilt disease complex in chickpea (Cicer arictum L.). Agric. Res. Rev., 69(3): 581 - 595.

Singh, K.N.; R.D. Prased and V.P.S. Tomar (1981). Response of French been to different levels of nitrogen and phosphorus in Nilgivi-Hills under rainfed condition. Indian Journal of Agronomy, 26: 101-102.

Soleman, N.K.; M.S. Mikhail; R.K. Harb and E.M. Khalil, (1988). Response of broad bean plants infected with Rhizoctonia solani to application of growth regulators and calcium. Egypt. J. Phytopathology, 20(1): 1-11.

Taha, S.M.; S.A.Z. Mahmoud; A.H. El-Damaty and A.M. Abdel-Hafez (1969). Activity of phosphate dissolving bacteria in Egyptian Soils. Plant and Soils, 31: 149-160.

Tripathi, R.D.; G.P. Srivastava; M.S. Misra and S.C. Pandey (1971). Protein content in some varieties of legumes. The Allah Abad Farmer, 16: 291-294. 